\title{
Close cardiac surveillance of patients treated with anthracyclines, also necessary after chemotherapy?
}

\author{
Leo H. B. Baur
}

Received: 13 October 2009/Accepted: 16 October 2009/Published online: 6 November 2009

(C) Springer Science+Business Media, B.V. 2009

In women, breast cancer is the second most common form of cancer and the leading cause of death caused by malignancy. Anthracyclines are well known chemotherapeutics for treatment of these tumors, other solid tumors and hematologic malignancies. As adjuvant treatment in breast cancer it improves survival and reduces recurrence [1]. Unfortunately, their clinical use is currently limited by the development of a progressive dose-dependent cardiomyopathy that irreversibly evolves toward congestive heart failure, which is usually refractory to conventional therapy [2]. Studies have reported that $10-26 \%$ of patients administered cumulative anthracycline doses above those, develop congestive heart failure, and that more than $50 \%$ of patients administered these doses will experience measurable functional impairment months to years after the end of therapy [3]. The pathophysiology of this cardiomyopathy is incompletely understood. Anthracyclines decrease $\beta$-andrenoceptor density in the cell membrane, increase the permeability of myocytes and alter the ultrastructure of myofibrils and actin networks [4]. Risk factors for anthracycline-induced cardiotoxicity include high

Editorial comment on the article of Appel et al. (doi:10.1007/ s10554-009-9518-2).

L. H. B. Baur $(\bowtie)$

Department of Cardiology, Atrium Medical Centre

Parkstad, University of Maastricht, Henri Dunantstreet 5, 6401 CX Heerlen, The Netherlands

e-mail: lbr01@atriummc.nl; lhbaur@yahoo.com cumulative anthracycline doses, high anthracycline dose intensity, and radiotherapy. Radiotherapy in patients with cancer treated with anthracyclines can exacerbate cardiac tissue damage. Cardiovascular complications of treatment may be observed during administration of the drugs, days to months following administration, or years to decades following exposure.

Several strategies have been developed to decrease the risk of cardiotoxicity while maintaining efficacy. These strategies include altered schedules of drug administration and adjunctive treatment with dexrazoxane. Unfortunately, none of these approaches have been successful, and patients remain at risk for the development of anthracycline cardiomyopathy, particularly with higher cumulative doses. Therefore careful serial monitoring is required for optimal medical management. Cardiomyopathy disease progression can be delayed in adults by using carvedilol or angiotensin-converting enzyme inhibitors [5, 6]. The permanent risk of cardiomyopathy by anthracyclines has made monitoring for the earliest evidence of cardiotoxicity necessary. In clinical practice monitoring is mostly performed using echocardiography or radionuclide angiography. The study of Appel et al. in the current issue of the International Journal of Cardiac Imaging shows that development of anthracycline toxicity is difficult to predict [7]. Although all patient in this study had a normal left ventricular function before receiving chemotherapy, $33 \%$ of the patients developed heart failure within a time period 
ranging from 2 months to 1.5 years. Left ventricular ejection fraction above $50 \%$ after chemotherapy appeared the best predictor of remaining asymptomatic. Diastolic variables did not predict heart failure at all. This study confirms the known evidence about anthracycline cardiomyopathy [8]. Because congestive heart failure can develop late, sometimes months to years after successful cancer treatment with anthracyclines, close surveillance of these patients with monitoring of left ventricular function by echocardiography or radionuclide imaging is needed. As this study shows, this is especially needed in those patients in whom ejection fraction decreased during chemotherapy. Heart failure treatment can than be initiated early and mortality improved.

\section{References}

1. Early Breast Cancer Trialists' Collaborative Group (1998) Polychemotherapy for early breast cancer: an overview of the randomised trials. Lancet 352:930-942
2. Mordente A, Meucci E, Silvestrini A, Martorana GE, Giardina B (2009) New developments in anthracyclineinduced cardiotoxicity. Curr Med Chem 16(13):1656-1672

3. Jensen BV (2006) Cardiotoxic consequences of anthracycline-containing therapy in patients with breast cancer. Semin Oncol 33(3 Suppl 8):S15-S21

4. Xu MF, Tang PL, Qian ZM, Ashraf M (2001) Effects by doxorubicin on the myocardium are mediated by oxygen free radicals. Life Sci 68:889-901

5. Lipshultz SE (2006) Exposure to anthracyclines during childhood causes cardiac injury. Semin Oncol 33(3 Suppl 8): S8-S14

6. Matsui H, Morishima I, Numaguchi Y, Toki Y, Okumura K, Hayakawa T (1999) Protective effects of carvedilol against doxorubicin-induced cardiomyopathy in rats. Life Sci 65(12): $1265-1274$

7. Appel JM, Jensen BV, Nielsen DL, Ryberg M, Zerahn B (2009) Systolic versus diastolic function variables during epiduricin treatment for breast cancer. Int $\mathrm{J}$ Cardiovasc Imaging. doi:10.1007/s10554-009-9518-2

8. Kremer LC, Caron HN (2004) Anthracycline cardiotoxicity in children. N Engl J Med 351(2):120-121 